American Journal of Applied Sciences 4 (8): 533-537, 2007

ISSN 1546-9239

(C) 2007 Science Publications

\title{
Introducing Radio-Opaque Radio-Lucent Graduated Root Canal Length Indicating Cones (GRCLIC) in Endodontics
}

\author{
Mohammad Rashid Shahidi Bonjar \\ School of Dentistry, University of Medical Sciences, Kerman, Iran
}

\begin{abstract}
Radio Opaque-Radiolucent Graduated Root-Canal Length Indicating Cone (GRCLIC) is a new innovative device being introduced for the first time in endodontics literature. GRCLIC can present more accurate measurement of the root canal length in conventional radiographs in endodontic and root canal treatment. Like many other dental and medical specialties, endodontics has evolved and changed over the years. The changes that have occurred in the past 10 years, however, have been of great magnitude and profundity. The microscope, ultrasonic units with specially configured tips, superbly accurate microchip computerized apex locators, flexible nickel-titanium files in rotary engines, and greater emphasis on microscopic endodontic surgery have totally changed the way endodontics and endodontic surgery are practiced. Every part of endodontic treatment is controlled by a measurement of the instrument's penetration depth into the canal, while the length is typically determined in millimeters. In this research, GRCLIC was used to enhance the more accurate root canal length determination. GRCLIC was made simply using a thin plastic or optic fiber graduated millimeter-wise with a heavy metal salt. The idea behind the GRCLIC technique was to present a new methodology which is actually a pre-exposure insertion of a simple graduated flexible ruler into the tooth canal instead of classic measurement methods. The main proved advantage of GRCLIC is decreasing mal-measurements which usually lead to treatment failures. GRCLIC enhances the success of endodontics treatment practices.
\end{abstract}

Key words: Graduated root canal length indicating cone (GRCLIC), endodontics treatment practices

\section{INTRODUCTION}

Working length (WL) is measured from a reference point (the reference point is the site on the occlusal or incisal surface from which measurements are made. This point is used throughout canal preparation and obturation) on the tooth's cavosurface that is within the clinician's field of view. It varies from the complete canal length to some arbitrarily determined point near the termination of the canal space. WL determines the extent of canal cleaning and shaping that will be accomplished. This measurement limits the penetration depth of subsequent instruments and determines the ultimate form of the shaping process. Cleaning and shaping can have no greater precision than the WL, hence it is extremely important to make an accurate determination. The objective of WL determination is to establish the length (distance from the apex) at which canal preparation and subsequent obturation are to be completed. Optimal length is 1 to $2 \mathrm{~mm}$ short of the apex, although this may vary slightly with different diagnoses $^{[1,2]}$. Procedures may be terminated 0 to $2 \mathrm{~mm}$ from the apex if the pulp is necrotic and 0 to $3 \mathrm{~mm}$ if the pulp is vital ${ }^{[3]}$. General methods used in determining the length of a canal include I) Radiographic and digital imaging (RVG): The most commonly used method of determining the length of a canal is radiographic ${ }^{[4]}$. The clinician starts by placing a file to an estimated length and then exposes a film. The location of the instrument tip is read from this film, and any necessary changes in length to reach the apical constricture are made. II) Electronic: Apex locators may be used to determine the canal length ${ }^{[5-9]}$. However, use of these devices does not result in precise determination of the apical constriction ${ }^{[10]}$. The unit leads are connected to a file that is inserted into the canal and to a lip clip that contacts the oral mucosa. The pulp is extirpated, the canal is irrigated and dried, and the attached file is inserted to the terminus. A dry canal and chamber eliminates ionic conduction, which can cause a premature indication that the apex has been reached. This is always necessary when the apex locator works on a resistance principle ${ }^{[11-13]}$. Apex locators are most helpful in placement of the first length-determining file. Without a locator, the working length must be estimated from a preoperative radiograph or other data. This estimation requires considerable clinical experience before it may be used dependably; therefore controlling the initial penetration depth with an apex locator 
removes the guess-work. One must be careful to avoid contaminating the file while connecting the electrical lead and inserting the measurement instrument. Without a radiograph mistakes are possible. For example, accidental passage through an accessory canal would indicate contact with the periodontal ligament; however, this length would be inappropriate. Electronic apex locators are especially useful when treating teeth with calcified pulp chambers. They test potential canal openings and detect a perforation before it is enlarged as a canal. The apex locator is superior to a radiograph in such situations. III) Tactile: The experienced clinician develops a keen tactile sense and gains considerable information from the passage of an instrument through a canal. Once radicular access has removed dentinal interference from the coronal third of a canal, the observant clinician can detect a sudden rise of resistance as a file approaches the apex ${ }^{[14]}$. A narrowing presses more tightly against the instrument, whereas curvature deflects the instrument and resists its passage. Both consume energy, and the sensitive hand can detect a sudden change in the pressure needed to maintain movement. The awareness of an apex can be enhanced by use of a file diameter that is equal or slightly larger than the constricture ${ }^{[15]}$. When a canal is constricted in its coronal two thirds, clinicians cannot discern apical anatomy with accuracy. IV) Paper point evaluation: Once the preparation is complete, a paper point may yield more than a dry cana ${ }^{[14]}$. After the canal is rendered dry, an additional paper point may be used to seek out apical moisture or bleeding. A bloody or moist tip suggests an overextended preparation or seepage of fluids into the canal. Further assessment of the apical preparation and working length should be made in this event. The point of wetness gives an approximate location to the actual canal end point. A wet or bloody point may also indicate that the foramen has been zipped or the apex perforated during preparation. V) Patient Response: Another fallacious belief is that if the pulp is necrotic or if canal preparation is complete, an unanesthetized patient will detect the file tip when it reaches and contacts the vital tissue at the apical foramen. Supposedly, the patient then signals this event with an "eye blink" or other pain response. There are problems with this approach also. First, the procedure is painful for the patient. Second, a necrotic pulp frequently contains vital inflamed tissue that extends several millimeters into the canal ${ }^{[16]}$. This tissue may be very sensitive and respond to instrument contact short of the apex. Third, a patient feels pain after canal preparation is complete from hydraulic pressure even though instruments do not reach the apical region.
In this research, Radio Opaque-Radiolucent Graduated Root-Canal Length Indicating Cone (GRCLIC) was developed to enhance the more accurate root canal length determination. The idea behind the GRCLIC technique is to present a new methodology which is actually a pre-exposure insertion of a simple graduated flexible ruler into the tooth canal instead of classic measurement methods. The main proved advantage of GRCLIC is decreasing mal-measurements which usually lead to treatment failures.

\section{MATERIALS AND METHODS}

Preparation of Graduated Cones: Fine glass fibers with approximate diameter of $0.1 \mathrm{~mm}$ and length of 30 $\mathrm{mm}$ were used. To make markings on the fibers, being both radio opaque and visible with naked eye, water soluble salts of heavy metals of lead and uranium were selected for the experiment. Saturated solution of lead acetate or uranyl acetate salts was prepared in $1: 1 \mathrm{v} / \mathrm{v}$ water-soluble ink: distilled water. Using a fine pointed brush, fine lines of marking solution was deposited on the fibers in $2 \mathrm{~mm}$ distances starting with the first marks from the tips and air dried using a hair drier. Graduated cones were then dipped in clear nail polish to retain a fine coating layer and dried before use. The coating prevents loss or distortion of graduation marks upon contact with interior surface of root canal or possible moisture. Tight endodontic conventional file rubber-stops were applied to each cone for two aims: a) GRCLIC calibration and b) to establish a reference point on the tooth surface.

In vitro access-cavity preparation: Extracted maxillary central teeth were used for the experiment. Following application of small portion (approximately $5 \mathrm{~mm}$ in diameter) of solid wax to root tips, teeth were fixed in a solid acrylic box (methyl metacrylate). Application of wax was to prevent the direct contact between rigid acrylic material and the apical foramen. Access cavity was made by a long tapered round end bur using a high torque hand piece.

GRCLIC calibration: For GRCLIC calibration, two points should be taken into consideration, first: the GRCLIC length (the length from GRCLIC tip to the rubber stop) must be partly longer (about $0-3 \mathrm{~mm}$ ) than the primary estimated tooth length (the primary tooth length can be estimated from the initial diagnostic radiographs, this length is definitely not accurate but it is almost near to the real length) and second: after GRCLIC is inserted throughout the canal till the tip has passed the apical foramen, the rubber stop edge (either 


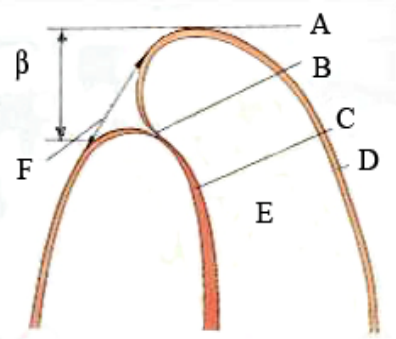

Fig. 1: Schematic root-terminal structure. $\beta(\approx 1 \mathrm{~mm})$ is approximate distance between foramen $(\mathrm{F})$ and root apex (A) which is considered as a correction factor for precision and is subtracted from the estimated working-length. B: Apical constriction, C: Root Canal, D: Cementum and E: Dentine

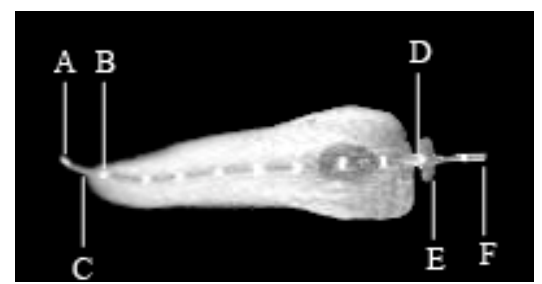

Fig. 2: Radio Opaque-Radiolucent Graduated Root-Canal Length Indicating Cone (GRCLIC) in position. Original radiograph is enhanced in Adobe Photoshop CS ver. 8.0 to perceive the details. A: First radio-opaque line exited root apex, B: Last radio-opaque line inside the canal. C: Apical foramen, D: Reference point, the first coronal radioopaque line outer the canal is calibrated with rubber stop edge (on reference point), E: Rubber stop, F: GRCLIC. The distance between $\mathrm{B} \& \mathrm{C}$ is the variant " $\mathrm{d}$ " in the formula $(\mathrm{d}=\mathrm{AB}-\mathrm{AC}$, which can visually be set as $0,0.5,1$ or $1.5 \mathrm{~mm}$ )

upper or lower) is adjusted to the first outer GRCLIC's line (nearest upper to the reference point). Afterwards the GRCLIC is pushed in the canal till the rubber stop touches the respective reference point (reference point is the site on occlusal or incisal surface from which measurements are made. This point is used throughout canal preparation and obturation on the tooth's cavosurface that is within the clinician's field of view). With these considerations, following radiography, then the GRCLIC is pulled out.

Radiography: The boxed tooth with inserted GRCLIC in was placed on a conventional PA radiographic dental film and the x-ray cone was adjusted vertically to expose the film. Radiography was performed according to the conventional procedures and subsequently, the film was developed regularly. The WL was then

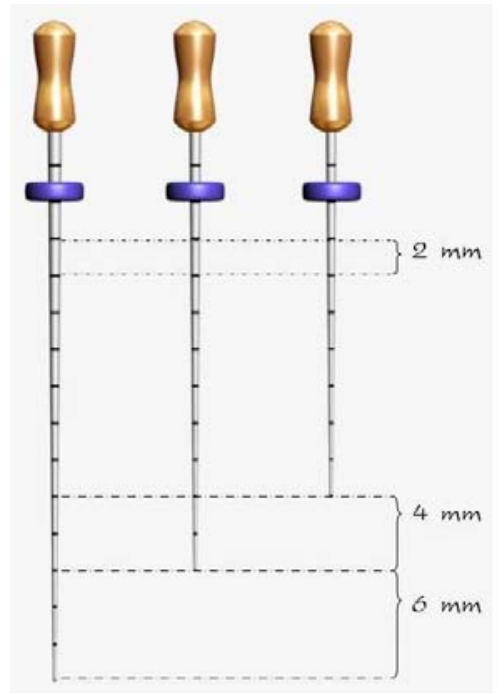

Fig. 3: Schematic image of GRCLIC in three sizes. Rubber stop is used to set the reference point

calculated via radiograph indicative of marked graduation of the canal.

Working length determination: WL determination was done in three steps as follows. Step 1) counting the number of radio opaque lines from the rubber stop (reference point) to the last opaque line in the canal on the developed film (N). Step 2) measuring the distance between the last opaque line in the canal to the radiographic apex (d, closest match of $0,0.5,1$ or 1.5 millimeters). Step 3) determining WL using the following formula: $\mathrm{WL}=2 *(\mathrm{~N}-1)+\mathrm{d}-\beta \Rightarrow W L=$ $2 N+d-3 . \beta(\approx 1 \mathrm{~mm})$ is approximate distance between foramen and root apex (Fig. 1) which is considered as a correction factor for precision and is subtracted from the estimated WL. If $\beta$ is not taken into calculation, radiographically working length ending 0 to $2 \mathrm{~mm}$ short of the radiographic apex provides, more often than expected, a basis for unintentional over-instrumentation. As indicated in Fig.2, if there are 9 opaque lines from the reference point in the canal $(\mathrm{N}=9)$ and there is about 0.5 millimeter distance between the last opaque line and the root apex $(\mathrm{d}=0.5 \mathrm{~mm})$, then the WL will be: $2 * 9+0.5-3=15.5 \mathrm{~mm}$. millimeters.

\section{RESULTS AND DISCUSSION}

Following working length determination with GRCLIC under an in vitro study: The actual size of working length was determined to be very precise as 

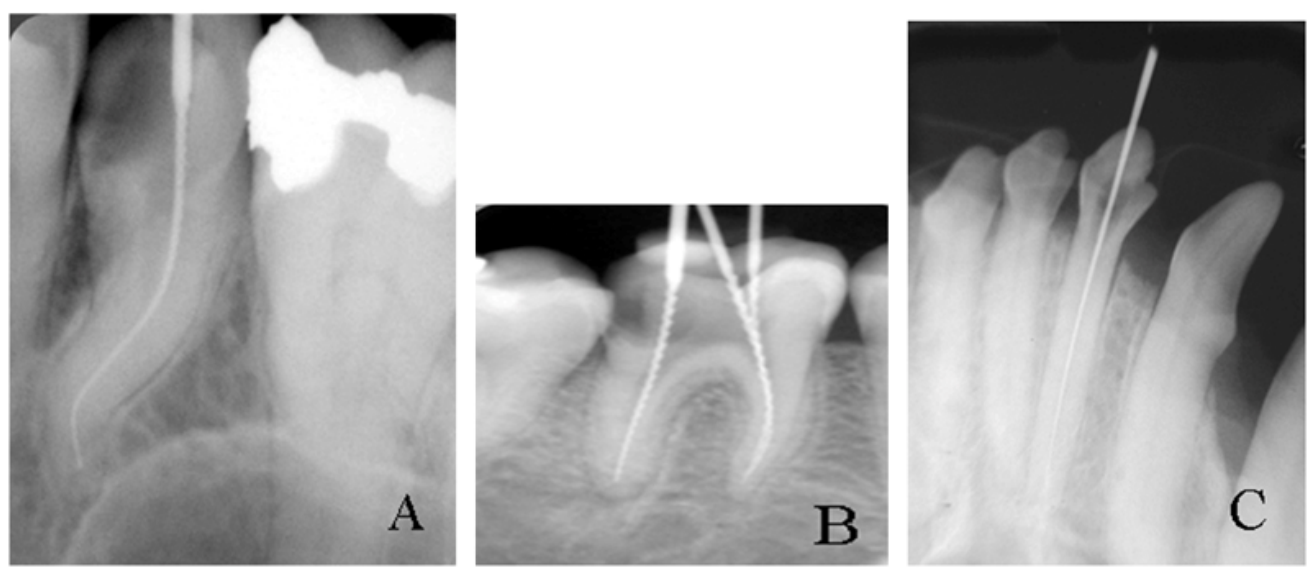

Fig. 4: Radiographic misjudgments which can be overcome by use of GRCLIC. Dilacerated root (A), radiographic distortions resulting from a wrong $\mathrm{x}$-ray tube angle or film bending lead to shortened-root images $(\mathrm{B})$ and elongated-root images (C). Use of GRCLIC in working-length measurement would not be affected with these situations since GRCLIC would behave as a flexible graduated ruler in canal

indicated in Fig. 2. The details of the figure are enhanced in Adobe Photoshop CS ver. 8.0 software to emphasize the expecting imaging in practice with industrialized or final manufactured GRCLICs. Schematic image of GRCLIC in three sizes is indicated in Fig. 3. The dimension is suggested to be as standard endodontic conventional K-file 20.

There are various clinical methods for WL determination, however, the available clinical methods used for WL measurements are also inaccurate ${ }^{[17]}$. Radiographs are common diagnostic devices usually deployed to determine the length of root canals in root canal therapy. Lengths will vary according to many factors; however, ideals are not always attainable. Apex location varies; these variations can be approximated but not usually determined using only a diagnostic radiograph ${ }^{[18,19]}$. However, this determination is an estimate, using average measurements of distances from the apical foramen to the true apex and from the apical constriction (or within the canal) to the apical foramen $^{[20]}$. Canals may exit on the root surface at a variable distance and position from the root tip and it is impossible to judge the position of apical foramina satisfactorily from radiographs. In progressive field of dental and medical specialties, endodontics has evolved and changed over the recent decades, including new treatment techniques and instruments for attainment of enhanced treatments. Adopting the microscope, ultrasonic units ${ }^{[21]}$ with specially configured tips, superbly accurate microchip computerized apex locators, flexible nickel-titanium files in rotary engines, digital imaging system (Radio Visio Graphy (RVG) $)^{[22]}$ and greater emphasis on microscopic endodontics surgery have enhanced the methodology of endodontics and endodontic surgery. These changes are bringing the specialty of endodontic practice into the twenty-first century with greater precision, fewer procedural errors, less discomfort to the patient, and faster case completions.

\section{CONCLUSION}

I believe that different techniques which are being used, studied, and advocated for determining WL, such as radiographic and tactile methods are not totally accurate or infallible. Because we cannot directly visualize the ends of root canals in vivo, length determination requires careful clinical assessment. Application of GRCLIC in conventional radiography in WL determination highly improves the accuracy of measurements while it decreases treatment faults such as: 1) over or under filing and obturation, 2) diagnostic radiographs produce two dimensional images of a three dimensional object, 3) technical errors of radiographic imaging of root canal such as low or high vertical X-ray cone angle, 4) radiographic magnification of the diverging central X-ray beam, 5) dilacerations / root curvatures and 6) film bending and distortion during exposure, leading to images as shortened or elongated roots (Fig. 4). In all of these cases, WL would not be mismeasured 
with using GRCLIC since it works as a flexible graduated ruler in canal. As in conventional procedures, in teeth with multiple canals, GRCLICs should be placed in all canals and a shifted single-view taken to minimize radiation.

GRCLIC has officially registered Iranian Patent Number of 33375 at 1384/09/09 under name of the author of this article.

\section{ACKNOWLEDGEMENTS}

This research is dedicated to Mr Ali Reza Afzali Pour and Mrs Fakhereh Saba, the founders of Universities in Kerman. I would like to thank Prof. Parirokh, Dr. Zarei, Dr. Faryabi, Dr. Mozaffari, Dr. Shahravan, Dr. Atai, Dr. Chamani, Dr. Eskandari Zadeh, Dr. Najafi, Dr. Motamedi, Dr. Mohammadi, Dr. Shahpasand Zadeh, Dr. Fayyaz, Dr. Ameri, Dr. Amini, Dr. Ghahremani, Dr. Navvabi, Dr. Sahranavard, Dr. Pour Eslami, Dr. Mosharafian, Dr. Farrokh Gisoo, Dr. Haghani, Dr. Hashemi, Dr. Rad, Dr. Momeni, Dr. Ansari and Dr. Tabari for their moral and scientific advices throughout my research and college studies.

\section{RERERENCES}

1. Kim, S., 2004. Modern endodontic practice, instruments and techniques. Dent. Clin. North. Am., pp:48:1-9.

2. Taintor, J., R. Biesterfeld and G. Valle, 1979. Termination of the root canal filling. Dent. Surv., 55: 54-59.

3. Wu, M.K., P. Wesselink and R. Walton, 2000. Apical terminus location of root canal treatment procedures. Oral Surg. Oral Med. Oral Pathol., 89: 99-103.

4. Woolhiser, G.A., J.W. Brand, M.M. Hoen, J.R. Geist, A.A. Pikula and F.E. Pink, 2005. Accuracy of film-based, digital, and enhanced digital images for endodontic length determination. Oral Surg. Oral Med. Oral Patol. Oral Radiol. Endod., 99: 499-504.

5. Haffner, C., M. Folwaczny, K. Galler and R. Hickel, 2005. Accuracy of electronic apex locators in comparison to actual length - an in vivo study. J. Dentistry, 8: 619-625.

6. Huang, L. 1987. An experimental study of the principle of electronic root canal measurement,.J. Endod., 13: 60-65.

7. Inoue, N, 1976. A study of audiometric devices for determining root length. Shikwa-Gakuo 76: 1121-1128.
8. Inoue, N, 1977. A clinico-anatomical study for the determining of root canal length by use of a novelty low frequency oscillation device. Bull. Tokyo Dent. Coll., 18: 71-90.

9. Venturi, M. and L. Breschi, 2005. A comparison between two electronic apex locators: an in vivo investigation. Int. Endod. J., 38: 36-45

10. Hoer, D. and T. Attin, 2004. The accuracy of electronic working length determination. Int. J. Endod., 37: 125-131.

11. McDonald, N.J., 1992. The electronic determination of working length. Dent. Clin. North Am., 36:293-307

12. McDonald, N.J. and E.J. Hovland, 1990. An evaluation of the apex locator Endo-cater. J. Endod., 16: 5-8.

13. Venturi, M. and L. Breschi. 2007. A comparison between two electronic apex locators: an ex vivo investigation. Int. Endod. J., Epub ahead of print.

14. Buchanan, L.S, 1991. Paradigm shifts in cleaning and shaping. J. Calif. Dent. Assoc., 23: 24-34.

15. Roane, J.B. 1991. Principles of preparation using the balanced force technique. In: Hardin J, Ed., 1991. Clark's clinical dentistry, Philadelphia.

16. Ricucci, D., 1998. Apical limit of root canal instrumentation and obturation. Part 1. Literature review, Int. Endod., 31: 384-390.

17. Gyorfi, A. and A. Fazekas., 2006. Problems with working length determination during endodontic therapy. Fogorv. Sz., 99: 153-159.

18. Burch J. and S. Hulen,1972. The relationship of the apical foramen to the anatomic apex of the tooth root. Oral Surg. Oral Med. Oral Pathol., 34: 262-266.

19. Olson, A.K., A. Goerig and R. Cavataio, 1991. The ability of the radiograph to determine the location of the apical foramen. Int. J., Endod, 24: 28-35.

20. Morris, A., S. Sylaras and M. Georgopoulou, 1994. Study of apices of human permanent teeth with the use of a scanning electron microscope. Oral Surg. Oral Med. Oral Pathol., 77: 172-176.

21. Ozan, A.U, 2005. Validating the roles of apex locators and sonic instrumentation in root canal therapy. Compend. Contin. Educ. Dent., 26: 6-10.

22. Marada, G. and G. Szabo, 2006. Clinical evaluation of digital imaging. Fogorv Sz., 99: 29-33. 